\title{
IMPACTO DEL MODELO DEL “JUEGO A LA SALUD” EN LOS ESTILOS DE VIDA SALUDABLES DE ESTUDIANTES DE PRIMARIA
}

\author{
Carlos Álvarez Bogantes \\ Escuela de Ciencias del Movimiento Humano y Calidad de Vida. \\ Facultad de Ciencias de la Salud, Universidad Nacional de Costa \\ Rica. \\ calvarez@una.ac.cr
}

\section{RESUMEN}

\begin{abstract}
El propósito de este artículo fue el de evaluar el efecto de una intervención interdisciplinaria en los hábitos de alimentación y la actividad física de niños y niñas de tercer grado. Diseño del estudio: Un diseño cuasi-experimental utilizado a través de 4 meses. Sujetos: tomaron parte en el estudio 27 niños y 32 niñas con edades promedio de 9.2 años. La intervención fue conducida por la maestra de grado, el maestro de cómputo, la maestra de educación para la vida en familia y el profesor de educación física, quienes proveyeron al grupo experimental con 4 clases académicas y de educación física en el área de nutrición y actividad física utilizó el programa Del "Juego a la Salud ". Resultados: Los niños y niñas en el grupo de intervención tuvieron un incremento significativo en el conocimiento, y en el tiempo reportado en actividad física $(p<.05)$ a través del reporte dirigido. Además, ellos mostraron un mejoramiento en el consumo de frutas y vegetales $(\mathrm{p}<.01)$. Con respecto a la actitud de cambio hacia la actividad física, las mujeres de la intervención mostraron cambios significativos. Conclusiones: El programa Del Juego a la Salud, mejoró el tiempo dedicado a la actividad física y al consumo de frutas y vegetales. Este estudio demostró ser práctico y que podría ser fácilmente incorporado al contexto educativo. Este programa puede mejorar el conocimiento en salud, en la actitud de cambio, y en las prácticas en nutrición y actividad física.
\end{abstract}

PALABRAS CLAVE: Intervención, actitud de cambio, actitud, actividad física, nutrición, niños, niñas.

\begin{abstract}
Objective: The purpose of th is article was to evaluate the impact of a room interdisciplinary health behavior intervention in diet y physical activity among children in grade 4. Study design: A cuasi-esperimental field trial, with a 1 intervention conducted from February 15 through May 31. Subjects: 27 boys y 32 girls with a 9.2 age mean, took part in the study. The intervention, taught by regular teacher, computer teacher, family education teacher y by the coordinator of the intervention the physical education teacher, provided the experimental group a nutrition y physical education health program called "From playing to health. Results: Children in the intervention group had a significantly greater health knowledge, y a selfreported physical activity $(\mathrm{P}<.05)$ Besides, they showed an improvement in fruit y vegetables intake $(\mathrm{P}<.01)$. Also, the girls from the intervention group had a significant change in the scale of the transtheoretical model of physical activity. Conclusions: This classroom-based program, improved children s physical time dedicated to physical activity y nutrition intake of fruit y vegetables; it is practical y fairly easy to incorporate into school context.. This program can improve health knowledge, attitude of change y habits (nutrition and physical activity).
\end{abstract}

KEY WORDS: Intervention, behavior change, Attitude, physical activity, nutrition.

\section{INTRODUCCIÓN}

Es sumamente difícil convencer a las autoridades educativas, padres y maestros de la importancia de establecer programas de promoción de la salud desde temprana edad dentro 
del currículo escolar. Esta tarea es incluso más difícil cuando las políticas educativas responden a esfuerzos no integradores de la realidad nacional; sin embargo, la evidencia abrumadora del inicio temprano de las conductas de riesgo de la enfermedad cardiodegenerativa en los niños, como lo son el sedentarismo y la obesidad, hace que los programas de prevención basados en la actividad física, sean una prioridad esencial en el sistema educacional y en el sistema de salud costarricense (Williams, 1994; Webber, Srinivasan, y Wettigney, 1991).

El seguimiento de las conductas de riesgo y de factores de riesgo de niños y niñas, sugiere que la promoción de conductas saludables a edades tempranas, podría tener efectos positivos a través de la vida. Varios estudios han mostrado resultados relativos en las intervenciones tradicionales para mejorar la actividad física y la nutrición en la población escolar (Simons-Morton, Parcel, Baranowski, Forthofer, O'Hara, 1991). Los estudios realizados en otros contextos, se han enfocado en poblaciones de alto riesgo, lo que ofrece un potencial limitado en la aplicación a una población de mayor espectro (Epstein, Coleman, y Myers, 1996). El desarrollo de programas de intervención en hábitos de actividad física y de nutrición, en niños y niñas, es una empresa importante y retadora, debido a que esta población sufre cada vez más los efectos de patrones de vida sedentarios y niveles de obesidad cada vez más altos (Burke, Frack, Milligan, y Taggart, 1998), propiciada no sólo por condiciones tecnológicas que estimulan estilos de vida inapropiados, sino también, por una cultura de inmovilidad, que eventualmente podría ponerlos en mayor riesgo de enfermedades degenerativas. (Rowly, 1998; Shephard, 1994).

Los estudios más importantes, han mostrado que los niños y las niñas más activos, presentan mejores niveles de aptitud física para la salud, presiones sanguíneas más bajas, niveles más bajos de HDL-colesterol y niveles de grasa corporal menores (U.S. Department of Health y Human Services, 1996), lo que disminuye el riesgo de enfermedades cardio-degenerativas para este grupo.

Sin embargo, los beneficios de la actividad física y de la buena alimentación en la prevención de enfermedades cardio-degenerativas en los niños y las niñas, no se han establecido contundentemente, esto debido a que las conductas inapropiadas para la salud, toman tiempo para que causen los efectos nocivos en el organismo o para que influencien la aparición de enfermedades crónicas, las cuales son desequilibrios orgánicos que se manifiestan en la vida adulta, en la mayoría de los casos (U.S. Department of Health y Human Services, 1996).

Algunos estudios reportan una relación positiva moderada entre inactividad física y los factores de riesgo coronario (Welk y Wood, 2000); pero otros han reportado resultados sin significancia estadística. En la actualidad, sigue creciendo la información relacionada con el proceso de inicio de aterosclerosis en edades más tempranas, lo que aumenta la importancia de la actividad física en el entorno escolar para poder impactar con mayor éxito los hábitos saludables de los niños. Aunado a lo anterior, la evidencia también muestra que los factores de riesgo coronario (hiperlipidemias, sedentarismo, obesidad y fumado) que propician la aterosclerosis y la enfermedad coronaria, se extienden dentro de la vida adulta 
aumentando las probabilidades del desarrollo de las enfermedades cardio-degenerativas (Harrell, Gansky, McMurray, Frauman, y Bradley, 1998).

Gran variedad de estudios han mostrado la efectividad del uso de la actividad física en intervenciones con población de niños con obesidad, la disminución de factores de riesgo de enfermedades degenerativas, el tratamiento de la diabetes mellitus y la disminución de niveles de sedentarismo en jóvenes (Epstein, Coleman, y Myers, 1996; Mahoney, Lauer, Lee, y Clarke, 1991; Whitaker, Wright, Pepe, Seidel y Dietz, 1997; Rippe y Hess 1998). Sin embargo, una gran cantidad de las intervenciones en niños y niñas en la promoción de estilos de vida saludables a nivel escolar han incrementado la cantidad de actividad física durante las clases de Educación Física; no así el incremento de actividad física en la vida cotidiana de los niños y niñas. (Stone, McKenzie, Welk y Booth, 1998; Simons-Morton, Parcel, Baranowski, Forthofer y O'Hara, 1991). Estos acercamientos biologistas, se contraponen a la intervención del Juego a la Salud, que pretende enseñar destrezas conductuales que ayuden a los niños y niñas a ser activos en diferentes entornos.

El propósito principal de este estudio fue el de evaluar el impacto de una intervención transversal, usando el programa "Del Juego a la Salud" en niños de tercer grado en las variables de conocimiento, actitud de cambio y en prácticas de actividad física y nutrición. Este artículo presenta los resultados de la intervención.

\section{METODOLOGÍA}

Sujetos. Esta investigación fue implementada con la participación de 27 niños y 32 niñas de cuarto grado de una escuela pública urbana de San José, durante 4 meses en el 2002, seleccionados al azar entre 6 grupos del nivel.

Instrumentos. Los niños y niñas participantes fueron evaluados y re-evaluados con: una prueba de conocimientos, reportes dirigidos de actividad física y nutrición y una escala de actitud de cambio. El mismo procedimiento fue realizado al grupo intervenido, siendo cada instrumento revisado con el niño y el padre. Adicionalmente, a los participantes se les consultó acerca de las barreras para realizar actividad física. Se hicieron mediciones de: grasa corporal con caliper marca Lange (Cambridge Scientific, Md.), los pliegues cutáneos fueron medidos tres veces en cada sitio y un promedio fue registrado. El índice de masa corporal fue calculado dividiendo el peso en kilos por el cuadrado de la estatura de cada participante. Todas las mediciones fueron realizadas a ambos grupos pre y post intervención, siguiendo el mismo orden en ambas mediciones.

Procedimientos. La intervención consistió en aplicar instrucción transversal, se utilizó los espacios de las materias de ciencias, computación, educación para el hogar, especialmente, las clases de educación física. Los niños y niñas recibieron de cuatro a seis lecciones semanales, en las cuales se usó material en enfermedades no transmisibles, sobre buena alimentación y sobre la importancia de realizar actividad física, siguiendo el modelo transteorético de cambio de conducta. En las clases de educación física, el profesor de 
educación física aplicó el programa "Del Juego a la Salud", que consistió en los siguientes momentos: en los primeros 5 o 10 minutos de la lección se hizo un recordatorio de los conceptos teóricos de las lecciones previas, seguido del calentamiento dirigido por alguno de los alumnos del grupo. Posterior a esto, el profesor dirigió uno o dos juegos que enfatizaron el concepto teórico de la semana. Una vez finalizada esta sección, el profesor, junto con los alumnos, realizaron una sesión de realimentación, donde los participantes recomendaron diferentes formas de realizar las actividades lúdicas. En la segunda parte de la clase, los alumnos practicaron diferentes destrezas físicas, que les ayudarían a ser más activos en el tiempo libre. La clase finalizó con la distribución de las actividades extra clase o con la toma de decisiones en cuento a las metas que cada uno se propondría en su camino para un estilo de vida saludable.

Los padres del grupo intervenido participaron en dos reuniones educativas sobre salud, con el fin de involucrarlos y capacitarlos en estilos de vida saludables.

Análisis estadístico. En todas las variables medidas se aplicó una T-Student para determinar las condiciones iniciales de ambos grupos. Posterior a esto, se ejecutaron análisis de varianza de tres vías (género x grupo x mediciones) para determinar el impacto de la intervención en las variables de conocimiento, prácticas en actividad física y nutrición. En el caso de la actitud de cambio un análisis de signos fue utilizado.

\section{RESULTADOS}

Como resultado de tratamiento, las variables fisiológicas no variaron en el post test, como se esperaba. Sin embargo, en la variable conocimiento (cuadro 1) hubo un incremento de un $45 \%$ en el grupo experimental, encontrándose una interacción positiva entre el pre y el post test del grupo experimental en comparación al control, lo que demuestra un efecto positivo de la intervención en esta variable.

\section{Cuadro 1}

PROMEDIOS Y DESVIACIONES ESTÁNDAR DE LA VARIABLE CONOCIMIENTO

\begin{tabular}{|c|c|c|c|c|}
\hline \multirow[t]{2}{*}{ Variable } & \multicolumn{2}{|c|}{ Grupo control } & \multicolumn{2}{|c|}{ Grupo experimental } \\
\hline & $\begin{array}{l}\text { Pre test } \\
\qquad(x \pm D E)\end{array}$ & $\begin{array}{l}\text { Post test } \\
(x \pm \text { DE })\end{array}$ & $\begin{array}{l}\text { Pre test } \\
(x \pm \text { DE })\end{array}$ & $\begin{array}{l}\text { Post test } \\
(x \pm \text { DE })\end{array}$ \\
\hline Conocimiento & 19.12 .53 & 19.532 .74 & 19.72 .29 & $323.55+*$ \\
\hline
\end{tabular}

+indica diferencias significativas entre mediciones (pre test vs. post test), $\mathrm{p}<0,05$

*(grupos por mediciones), $\mathrm{p}<0,05$

Con respecto a la variable de actitud de cambio, se encontró una interacción positiva entre las niñas del grupo experimental y control, en el pre test y post test. Esto demuestra un efecto positivo en la actitud de cambio hacia la actividad física de las mujeres del grupo experimental $(<.05)$. 
Cuadro 2

PROMEDIOS Y DESVIACIONES ESTÁNDAR DE LAS VARIABLES GASTO CALÓRICO (CAL) Y TIEMPO EN GRUPO EXPERIMENTAL

\begin{tabular}{|c|c|c|c|c|}
\hline Variable & \multicolumn{2}{|c|}{ Mujeres } & \multicolumn{2}{|r|}{ Hombres } \\
\hline & $\begin{array}{l}\text { Pre test } \\
(x \pm D E)\end{array}$ & $\begin{array}{r}\text { Post test } \\
(x \pm D E)\end{array}$ & $\begin{array}{l}\text { Pre test } \\
(x \pm D E)\end{array}$ & $\begin{array}{l}\text { Post test } \\
(x \pm \text { DE })\end{array}$ \\
\hline $\begin{array}{c}\text { Gasto calórico } \\
\text { Tiempo (m) }\end{array}$ & $\begin{array}{cc}1154 & 84.13 \\
15.371 & 2.450\end{array}$ & $\begin{array}{rr}1282 & 62.20 \\
24.522 & 3.446\end{array}$ & $\begin{array}{cc}1331 & 73.75 \\
24.34 & 4.032\end{array}$ & $\begin{array}{l}143547.67 \\
27.7853 .423\end{array}$ \\
\hline
\end{tabular}

*indica una interacción significativa (grupos $\mathrm{x}$ mediciones), $\mathrm{p}<0,05$

+indica diferencias significativas entre mediciones (pre test vs. post test), $\mathrm{p}<0,05$

El incremento porcentual del grupo experimental fue de 68\%. En el análisis de la variable de alimentación, como se aprecia en el cuadro se encontraron interacciones positivas entre los grupos experimental y control en el pre test y post test, lo que demuestra un efecto positivo de la intervención (<.05).

Al analizar la variable de incremento de tiempo (cuadro 2) dedicado a la actividad física moderada, se encontró que hubo interacciones positivas entre el grupo experimental y control en el pre test y el post test, notándose cambios mayores en las mujeres del grupo experimental en relación con los hombres del mismo grupo (<.05).

Cuadro 3

PROMEDIOS Y DESVIACIONES ESTÁNDAR DE LA VARIABLE ALIMENTACIÓN

\begin{tabular}{c|ccccc}
\hline Variable & \multicolumn{3}{|c}{ Grupo control * } & \multicolumn{2}{c}{ Grupo experimental* } \\
\hline & $\begin{array}{l}\text { Pre test } \\
(\mathrm{x} \pm \mathrm{DE})\end{array}$ & $\begin{array}{l}\text { Post test } \\
(\mathrm{x} \pm \mathrm{DE})\end{array}$ & $\begin{array}{c}\text { Pre test } \\
(\mathrm{x} \pm \mathrm{DE})\end{array}$ & $\begin{array}{c}\text { Post test } \\
(\mathrm{x} \pm \mathrm{DE})\end{array}$ \\
\hline Alimentación & $\mathbf{1 . 6 1} .46$ & $\mathbf{1 . 0 6} .24$ & $\mathbf{1 . 5 1} .56$ & $\mathbf{2 . 8 7}$ & $.95+$ \\
\hline
\end{tabular}

+indica diferencias significativas entre mediciones (pre test vs. post test), $p<0,05$

*(grupos por mediciones), $\mathrm{p}<0,05$

\section{DISCUSIÓN}

Este estudio evaluó el efecto de una intervención de 16 semanas en niños y niñas de cuarto grado de una escuela pública de Costa Rica, siguiendo el programa Del Juego a la Salud, el cuál recomienda el uso de educación para la salud y actividad física siguiendo los elementos básicos del Círculo Analítico, en unión con los procesos del modelo Transteorético de cambio de conducta. Los resultados de este estudio muestran que es la primera intervención conocida en el ambiente costarricense educativo, que evidencia, que es posible incrementar la participación en actividad física en los niños a partir del ambiente educativo. Después de estar expuestos al Programa del Juego a la Salud, los niños y niñas 
participantes, reportaron incrementos de un $20 \%$ y un $48 \%$ respectivamente, de actividad física moderada en su vida cotidiana. Este estudio muestra que es posible implementar programas a nivel escolar que promuevan no solo la actividad física en los niños y niñas, pero que también contribuyan al objetivo de salud pública de incrementar los niveles de movimiento entre nuestros niños.

Es evidente, que este estudio fue único, debido a que fue conceptualizado como una intervención en salud pública que se concentró en niños y niñas, que atendieron a un nivel escolar determinado. A diferencia de muchos estudios conocidos, que han reclutado sujetos para participar en el estudio (Pate et al, 2003; Gutin, Barbeau y Owens, 2002). Los hallazgos de este estudio mostraron un incremento mayor en actividad física moderada en el grupo de niñas, en comparación a los niños. Aunque este cambio no alcanzó los niveles que se recomiendan para los niños y niñas (Corbin y Pancrazi, 1996; Pancrazi, Corbin y Welk, 1996), si es importante, ya que indica que si fuera aplicado a la población de niños y niñas del país, se estaría en camino de lograr los recomendado en el ámbito de salud para esa población.

Gran cantidad de escuelas en nuestro país ofrecen la materia de educación física; sin embargo, son programas tradicionales que fallan en enseñar destrezas conductuales que puedan ayudar a los estudiantes a ser activos en la vida cotidiana (Centers for Disease Control and Prevention, 1998).

Todo hace indicar, que la intervención realizada en esta investigación fue más efectiva cuando se compara con las intervenciones, como Program Lifestyle Education for Activity (Ward et al, 2006) o la del proyecto Coordinated School Health Program (CSHP) Model (Felton et al, 2005), los cuales duraron más de un año. Estos estudios reportaron buenos resultados cualitativos en la participación de las niñas; sin embargo, este estudio mostró más impacto en la variable de actividad física en ambos sexos, en comparación a los estudios anteriores. En el estudio de Harrel et al. (1996) llamado Salud Cardiovascular de los Niños, lógicamente se obtuvieron buenos resultados en el ámbito de actividad física en los participantes, pero fue un estudio más intenso, que se basó en actividad física todos los días y clases magistrales durante un año.

Se observa, que los resultados del estudio sobre las otras variables, tuvieron un efecto positivo en las variables de conocimiento, actitud de cambio y prácticas en nutrición y tiempo dedicado a la actividad física, con respecto al grupo control. requiere de aumentos, aunque estos sean mínimos. Aunque los resultados de esta investigación no posibilitan mostrar una tendencia hacia la inactividad física a través del tiempo, si se podría mencionar que estos datos reflejan un alto grado de conducta sedentaria en el ámbito de las niñas, el cuál coloca a esta población con una situación de riesgo a temprana para el desarrollo de enfermedades cardio-vasculares, obesidad y diabetes (Epstein, Saelens, Myers y Vito, 1997).

La obesidad ha aumentado dramáticamente entre los niños durante los últimos tiempos. Las autoridades en salud y en educación han identificado que la promoción de la actividad 
física es un estrategia indispensable en el ataque de este problema (American Academy of Pediatrics, 2002). En este estudio, los resultados del análisis de grasa corporal pos-test, no mostraron cambios significativos en los niños y niñas de este estudio. Los resultados no solo sobrepasan los valores apropiados para la salud conocidos, sino que también son superiores a los valores del estudio nacional realizado por Fernández, Ulate, Romero, y Murillo (1996), lo que reforzó el conocimiento que los cambios en grasa corporal requieren de intervenciones más prolongadas (Mahoney et al., 1991).

Además, algunas otras explicaciones de los resultados obtenidos podrían ser que no se ofreció un manejo más amplio del control de ingesta calórica. Como se puede apreciar con los cambios en las prácticas alimenticias, se mantuvo la base de arroz, aceite, azúcar y en alguna medida frijoles después de la intervención. También se encontró un incremento en la ingesta de frutas y vegetales, en una forma amplia. Adicionalmente, se notó una disminución en la ingesta de embutidos, lo que significa una disminución en la ingesta de grasa saturada, según las proporciones recomendadas por el Ministerio de Salud (1996). Sin embargo, cambios en la compasión corporal, posiblemente, requerirán al menos una intervención durante la primaria. Estudios futuros deberán tratar de darle respuesta a esta disyuntiva.

Aunque las clases de educación física y el rol del educador físico fueron esenciales, la información de este estudio sugiere, que el efecto total no se le puede achacar expresamente a la participación en las clases de educación física, sino a la intervención transversalintegral que ejecutó este estudio.

En el caso del Programa del Juego a la Salud, se decidió utilizar una intervención que no implicara inclusión de nuevos elementos en el currículo, por lo que lo que se realizó fue un reordenamiento de los contenidos en salud que los educadores ya realizaban. El articular los conocimientos en salud permitió un refuerzo constante entre cada uno de los contenidos cubiertos. El único que asumió un rol más activo, fue el educador físico, el cuál coordinó y reforzó el trabajo de los otros educadores, utilizando el formato del juego a la Salud. Aunque los padres jugaron un papel de facilitadores o modificadores del entorno en esta propuesta, correspondió a los niños, con sus nuevas actitudes, destrezas y conocimientos propiciar un cambio en su hogar. Se debe recalcar el hecho de que este programa obtuvo cambios radicales en conocimiento, actitud de cambio, hábitos de movimiento y prácticas de alimentación en los participantes, siendo las niñas las que mayores cambios tuvieron.

\section{CONCLUSIONES}

Gran cantidad de conductas relacionadas a la salud son formadas en la niñez, lo que sugiere que la escuela primaria ofrece un punto crucial para intervenir. Se necesita identificar programas de intervención que no solo sean efectivos, sino que también respeten la idiosincrasia de los niños y niñas escolares de Costa Rica, que efectivamente mejoren las conductas en salud, especialmente las relacionadas con la nutrición y la actividad física, con el afán de reducir los factores de riesgo cardiovasculares. Este estudio es el primer estudio conocido que interviene a niños escolares con gran éxito a un costo ínfimo. 
Este programa demostró que el usar el modelo transteorético de cambio de conducta se logró un incremento sustancial en las proporciones de alimentos diarios de los estudiantes del grupo experimental, especialmente en lo referente a frutas y vegetales. En relación con el conocimiento, este mejoró sustancialmente, al igual que el tiempo dedicado a la actividad física, aunque en esta última variable, no se lograron los valores recomendados para la salud. En la variable de cambio de conducta se lograron cambios prometedores y exitosos en el uso del modelo utilizado, fundamentalmente en las mujeres.

Se sugiere, sin embargo, que para lograr resultados permanentes en las variables estudiadas y en el perfil de riesgo cardiovascular, se recomienda intervenir una vez al año durante los cinco años de la primaria y la secundaria, lo que deja un gran reto a los promotores en salud de Costa Rica, con respecto al desarrollo de insumos que puedan ser utilizados en todos los niveles educativos, pero sobre todo la lucha que tiene que ver con la inclusión de esos elementos en el currículo escolar.

\section{REFERENCIAS}

American Academy of Pediatrics. Physical fitness and activity in schools. Pediatrics. 2000;105:1156-1157.

Burke, V., Frack, R., Milligan, A., y Taggart, A. (1998). A controlled trial of health promotion program. Journal of Pediatrics. 16, 840-848.

Centers for Disease Control and Prevention. (1998). Guidelines for school and community programs to promote lifelong physical activity among young people. Morbidity and Mortality Weekly Report (MMWR). ;46:1-36.

Corbin, C. y Pancrazi, R. (1996). How much physical activity is enough? Journal of Physical Education, Recreation \& Dance (JOPERD): 67, 33-39.

Epstein, L., Coleman, K. y Myers, M. (1996). Exercise in treating obesity in children and adolescents. Medicine y Science in Sports y Exercise. 28, 428-435.

Epstein, L., Saelens, B., Myers, M. and Vito.D. (1997). Effects of decreasing sedentary behaviors on activity choice in obese children. Health Psychology. 16, 107-113.

Fernández A, Ulate G, Romero M, y Murillo S. (1996). Factores de riesgo cardiovascular en estudiantes de 17-19 años de la Universidad de Costa Rica. Documento Técnico, Facultad de Medicina, Universidad de Costa Rica.

Felton G, Saunders RP, Ward DS, Dishman RK, Dowda M, Pate RR. (2005). Promoting physical activity in girls: a case study of one school's success. Journal of School Health. 75(2):57-62. 
Harrell, S., Gansky, S., McMurray, R., Frauman, A., y Bradley, C. (1998). School based interventions improve heart health in children with multiple cardiovascular disease risk factors. Pediatrics. 102, 371-380.

Gutin B, Barbeau P, Owens S, (2002). Effects of exercise intensity on cardiovascular fitness, total body composition, and visceral adiposity of obese adolescents. The American Journal of Clinical Nutrition. 75:818-826.

Mahoney, L., Lauer, R. ., Lee, J., y Clarke, W. (1991). Factors affecting tracking of coronary heart disease risk factors in children. The muscative study. Journal of School Health. 61, 224-227.

Ministerio de Educación Pública (1997). Evaluación de la salud física de estudiantes de la educación general básica. San José, Costa Rica.

Ministerio de Salud (1996) Instituto Costarricense de Investigación y Enseñanza en Nutrición y Salud. Encuesta de Micronutrientes. Costa Rica: MS/Inciensa.

Pancrazi, R., Corbin, C. y Welk, G. (1996). Physical activity for children y youth. Journal of Physical Education, Recreation \& Dance (JOPERD). 67, 38-43.

Pate RR, Saunders RP, Ward DS, Felton GM, Trost SG, Dowda M. (2003). Evaluation of a community-based intervention to promote physical activity in youth: lessons from Active Winners. The American Journal of Clinical Nutrition. 17:171-182.

Rippe, J., y Hess, S. (1998). The role of physical activity in the prevention y management of obesity. Journal of the American Dietetic Association. 98, S31.

Rowly, T. (1998). The biological basis of physical activity. Medicine y Science in Sports y Exercise. 30, 392-399.

Simons-Morton BG, Parcel GS, Baranowski T, Forthofer R, O'Hara NM. (1991). Promoting physical activity and a healthful diet among children: results of a schoolbased intervention study. American Journal of Public Health. 81:986-991.

Stone EJ, McKenzie TL, Welk GJ, Booth ML. (1998). Effects of physical activity interventions in youth: review and synthesis. American Journal of Preventive Medicine. 15:298-315.

Shepard, R., (1994). Physical activity y child health. Sport Medicine. 1, 205-233. 
U.S Department of Health y Human Services. (1996). Physical activity y health: reports of the Surgeon General. Atlanta, GA: U.S Department of Health y Human Services, Centers for Disease Control y Prevention.

Ward DS, Saunders R, Felton GM, Williams E, Epping JN, Pate RR. (2006). Implementation of a school environment intervention to increase physical activity in high school girl. Health Education Research. 21(6):896-910.

Webber, L., Srinivasan, y S.,Wettigney, W. (1991). Tracking of serun lipids y lipoproteins from childhood to adulthood. American Journal of Epidemiology. 133, 884-899.

Welk, G. (2000). Measurement issues in the assessment of physical activity in children. Research Quarterly for Exercise and Sport (RQES). 71, 59-73.

Welk, G. y Wood, K. (2000). Physical activity assessments in physical education. Journal of Physical Education, Recreation \& Dance (JOPERD). 71, 30-40.

Whitaker, R., Wright, J., Pepe, M., Seidel, K. y Dietz, W. (1997). Predicting obesity in young adulthood from childhood y parental obesity. The New Engly Journal of Medicine. 337, 869-873.

Williams, C. (1994). Coronary heart disease prevention in childhood part I: background y rationale. Medicine, Exercise, Nutrition y Health. 3, 194-205.

Fecha de recepción: 21 de mayo del 2008.

Fecha de aceptación: 4 de octubre del 2011.

Fecha de publicación: 31 de diciembre del 2012. 